\title{
Evaluation of the Effect of Combination Therapy on Treatment of COVID-19: A Cohort Study
}

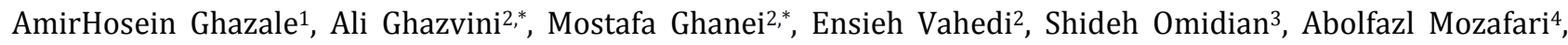
Mohammad Rezapour ${ }^{1}$, Nafiseh Rastgoo ${ }^{5}$, Fatemeh Movaseghi ${ }^{6}$, Fateme Mansouri ${ }^{1}$, MohammadAli Zohal ${ }^{3}$, Maryam Gheraati ${ }^{3}$, Seyed Hassan Saadat ${ }^{7}$, Hassan Goodarzi ${ }^{10}$, Mohammad Gholami Fesharaki ${ }^{8}$, AmirMohammad Dehghan Banadkooki ${ }^{1}$, Shahrzad Saloo ${ }^{1}$ and Hesamodin Salou ${ }^{9}$

${ }^{1}$ Student Research Committee, Baqiyatallah University of Medical Sciences, Tehran, Iran

2 Chemical Injuries Research Center, Systems Biology and Poisoning Institute, Baqiyatallah University of Medical Sciences, Tehran

${ }^{3}$ Metabolic Diseases Research Center, Research Institute for Prevention of Non-Communicable Diseases, Qazvin University of Medical Sciences, Qazvin, Iran

${ }^{4}$ Department of Medical Science, Qom Branch, Islamic Azad University, Qom, Iran

${ }^{5}$ Student Research Committee, Qazvin University of Medical Sciences, Qazvin, Iran

${ }^{6}$ Department of Medical Science, Qom Branch, Islamic Azad University, Qom, Iran

7 Behavioral Sciences Research Center, Lifestyle Institute, Baqiyatallah University of Medical Sciences, Tehran, Iran

${ }^{8}$ Department of Biostatistics, Faculty of Medical Sciences, Tarbiat Modares University, Tehran, Iran

${ }^{9}$ School of Veterinary Medicine, Semnan University, Semnan, Iran

${ }^{10}$ Trauma Research Center, Baqiyatallah University of Medical Sciences, Tehran, Iran

* Corresponding author Ali Ghazvini, Chemical Injuries Research Center, Systems Biology and Poisoning Institute, Baqiyatallah University of Medical Sciences, Tehran. Tel: 09121755664; Email: Qazvinia@gmail.com

Mostafa Ghanei, Chemical Injuries Research Center, Systems Biology and Poisoning Institute, Baqiyatallah University of Medical Sciences, Tehran. Tel: 09123209673; Email: mghaneister@gmail.com

Received 2021 March 05; Revised 2021 April 17; Accepted 2021 May 20.

\begin{abstract}
Background: COVID-19 is a new disease for which a definitive treatment has not yet been proposed.

Objectives: The present study aimed to investigate the effect of combination therapy on the treatment of COVID-19 due to the importance of finding an appropriate treatment for this epidemic disease.

Methods: This two-center cohort study included 175 confirmed COVID-19 inpatients at two medical centers designated for the treatment of COVID-19 patients in Qom and Qazvin, Iran. In this study, four different groups of drug regimens were studied which included G1 (azithromycin, prednisolone, and naproxen), G2 (lopinavir/ritonavir, azithromycin, naproxen, and prednisolone), G3 (hydroxychloroquine, azithromycin, naproxen, and prednisolone), and G4 (levofloxacin, vancomycin, hydroxychloroquine, and oseltamivir). It should be noted that G1, G2, G3, and G4 treatment regimens were used on 48, 39,30, and 77 patients, respectively.

Results: The study participants included 175 confirmed COVID-19 patients with mean \pm SD age of $58.9 \pm 15.1$ years, out of whom 80 (46\%) patients were male and the rest were females. The results indicated that the hospital stay period was significantly shorter in the G1 compared to other groups (G1:5.9 $\pm 2.4, \mathrm{G} 2: 8.1 \pm 4.2, \mathrm{G} 3: 6.3 \pm 1.7$, and G4: $6.4 \pm 2.9$; [P-value=0.008]). It should be noted that pulse rate, oxygen saturation, hemoglobin, and platelet count (PLT) changed significantly during the study in four treatment groups; however, a significant change in temperature, creatinine, and white blood cell (WBC) was observed only in G3, G4, and G1 groups, respectively. The number of ICU admissions and deaths were not statistically significant among the patients who received the four treatment regimens $(\mathrm{P}=0.785)$. Based on the results, the history of ischemic heart disease, baseline oxygen saturation, WBC, neutrophil, lymphocyte count, and C-reactive protein (CRP) are the risk factors for the prolonged hospital stay in COVID-19 patients.

Conclusion: The obtained results in this study indicated that the combination of azithromycin, prednisolone, and naproxen is the most
\end{abstract} effective regimen for the treatment of COVID-19, compared to three other combination treatment regimens.

Keywords: Anti-inflammatory drugs, Antiviral drugs, Combination therapy, Corticosteroid, COVID-19, Immunomodulators drugs

\section{Background}

Coronavirus disease 2019 (COVID-19) is a new virus disease that started in Wuhan, China, and was quickly transformed into a pandemic. This pandemic has posed an unprecedented impact on the healthcare system due to the high spread of the disease and its mortality rate (1).

Nowadays, the lack of a specific, effective, and proven standard treatment for COVID-19 disease is one of the serious public health challenges worldwide. Many aspects of COVID-19 disease including demographic characteristics of patients, clinical features, and biological abnormalities, as well as the radiological and pathological patterns of the disease have been described so far. Moreover, many studies have addressed different therapeutic strategies for the management of the disease.

The proposed medicines for the treatment of COVID-19 included Kaletra (lopinavir-ritonavir), remdesivir, oseltamivir, ribavirin, sofosbuvir (2-5), immunomodulators, such as chlorine and hydroxychloride (6-7) as well as anti-inflammatory medications, such as corticosteroids (8-10) or their combination (11).

The main COVID-19 management strategy has its focus on antiviral treatments with Kaletra (lopinavirritonavir), remdesivir, oseltamivir, ribavirin, sofosbuvir (2-5), immunomodulators, such as chlorine and hydroxychloride $(6,7)$ and anti-inflammatory 
therapies, such as corticosteroids (8-10) or their combination, owing to the two-phase pathogenesis of the disease (11).

Antiviral therapies are effective in the treatment of such diseases as Middle East respiratory syndrome coronavirus, severe acute respiratory syndrome coronavirus, and influenza. However, the effectiveness of these medications in the treatment of COVID-19 has not been widely approved, even though evidence indicates the decreasing pattern of viral load over time. The other management strategies are focused on anti-inflammatory therapies, such as the use of corticosteroids, particularly in patients with organizing pneumonia patterns $(8,9)$.

In general, the administration of corticosteroids has been recommended for critically ill patients suffering from COVID-19 (10). A combination of antiviral drugs with corticosteroids has also been suggested which may effectively decrease viral load and treat inflammation in the patients.

\section{Objectives}

Due to the importance of COVID-19 treatment, the present study aimed to investigate the combination therapy on the treatment of this disease.

\section{Methods}

This cohort study included 175 confirmed COVID19 patients in the age range of 25-95 years who were hospitalized at two medical centers designated for the treatment of COVID-19 in Qom and Qazvin, Iran, from March 17 to April 29, 2020. All the COVID-19 patients in this study were diagnosed according to the WHO interim guidelines. The medical records of all patients were obtained from the hospital data and the death of any patient was recorded as an outcome. The study was approved by the Medical Ethics Committee of Baqiyatallah University of Medical Sciences (IR.BMSU.REC.1399.001).

According to the data obtained from the hospitals, each patient received one of the four combination therapy regimens and was consequently assigned to one of the four groups of 1,2,3, and 4. Medicines applied on patients in Group1 included azithromycin (250 mg daily), prednisolone (25 mg/daily), and naproxen (250 $\mathrm{mg}$ BD). Medication in Group 2 included lopinavir/ritonavir (200/50 mg tablets two times/12 h), azithromycin (250 mg daily), naproxen (250 mg BD), and prednisolone (25 mg daily). Patients in Group 3 took hydroxychloroquine (250 $\mathrm{mg}$ tablets two times/12 h), azithromycin (250 mg daily), naproxen (250 mg BD), and prednisolone (25 mg daily). Medicine regimen in Group 4 consisted of meropenem (1gr/8h), levofloxacin (500 mg/day), vancomycin (1gr/12h), hydroxychloroquine (200 $\mathrm{mg} / 12 \mathrm{~h}$ ), and oseltamivir (75 mg/12 h).
Inclusion criteria in this study included 1) Diagnosis of COVID-19 based on approved diagnostic tools, such as real-time RT-PCR (throat-swab specimens) or chest CT scan according to the WHO interim guidelines which included symptoms of typical coronavirus pneumonia (i.e., patchy infiltration and focal unilateral to ground-glass opacity, ill-defined margins, smooth or irregular interlobular septal thickening, air bronchogram, crazy-paving pattern, and thickening of the adjacent pleura) (23-14), 2) Oxygen saturation (SPO2) $\leq 93,3$ $16 \leq$ Age $\leq 100,3)$ Patients with controlled diabetic status, no history of gastrointestinal bleeding, nonpregnant and non-lactating mothers, 4) Patients with no history of immunosuppressive therapy or chemotherapy in the past month. However, patients with high missing data in the medical records were excluded from the study.

In this study, the patients' demographic data, history of past diseases, and laboratory findings (i.e., white blood cell count (WBC), neutrophil, lymphocyte, hemoglobin, platelet count (Plate), Creactive protein (CRP), creatinine) were obtained. Moreover, the vital signs of the patients were monitored which included temperature, respiratory rate (RR), pulse rate (PR), systolic blood pressure (SBP), diastolic blood pressure (DBP), and resting stable oxygen saturation (5 min after disconnecting). Eventually, symptoms, such as cough, fever, dyspnea, weakness and lethargy, headache, dizziness, anorexia, and body pains were extracted from the medical records of hospitalized patients.

\subsection{Statistical Analysis}

The data were analyzed using R software (version 3.2 for Windows) based on non-missing values through the Kruskal-Wallis Test, analysis of variance (ANOVA), analysis of covariance (ANCOVA), and Chisquare test (for comparing demographic and baseline variables among the four combination regimens). Qualitative and quantitative variables were reported in percentages and mean $\pm S D$, respectively. A p-value less than $0.05(\mathrm{P} \leq 0.05)$ was considered statistically significant.

\section{Results}

Initially, the medical records of 490 patients were randomly selected out of 1270 hospitalized patients. Eventually, the medical records of 175 patients were analyzed (Figure 1).

Females $(n=95,54 \%)$ constitutes the majority of COVID-19 patients $(n=175)$ in this study. The mean \pm SD age of patients was estimated at $58.94 \pm 15.14$ years with the age range of 25-95 years. Table 1 presents the distribution of demographic characteristics, baseline variables, comorbidities, clinical features, and laboratory findings of four treatment groups. The major comorbidities at the time of admission included 
hypertension $(27 \%)$, ischemic heart disease $(9 \%)$, and hypothyroidism (5\%). No significant difference was observed in variables of four treatment groups except for variations in gender $(\mathrm{P}<0.001)$, body temperature $(\mathrm{P}=0.001)$, CRP $(\mathrm{P}=0.038)$, and pulse rate $(\mathrm{P}=0.015)$.

Table 2 presents the distribution of discharge status and hospital stay in four treatment groups. The number of ICU admissions and deaths were not found to be statistically significant in the four treatment groups $(\mathrm{P}=0.785)$. In addition, hospital stay was found to be significantly lower in Group1 compared to other groups $(\mathrm{P}=0.008$; Table 2). The hospital stay was just about two days shorter in Group1, compared to Group 2, and 0.5 days shorter than that in Group 3 and 4 .

As can be seen in Table 3, PR, oxygen saturation, hemoglobin, and PLT have significantly changed during the study in four treatment groups; however, temperature, creatinine, and WBC significantly changed in Group 3, 4, and 1, respectively.

The history of ischemic heart disease was more prevalent among the patients discharged from ICU $(\mathrm{n}=11)$ and those who died $(\mathrm{n}=8)$ in this study (9\% vs $26 \%$, P-value $=0.026$ ). Based on the results, baseline SPO2 (88.1 \pm 6.78 vs. 84.87 \pm 7.4$)$, WBC (7.41 \pm 9.09 vs. $13.03 \pm 14.2, \quad \mathrm{P}=0.013)$, neutrophil $(72.36 \pm 12.7$ vs. $80.23 \pm 6.64, \mathrm{P}$-value $=0.022$ ), lymphocyte count $(16.18 \pm 6.04$ vs. $16.18 \pm 6.04$, P-value $=0.038)$, and CRP $(21.65 \pm 17.26$ vs. $29.11 \pm 12.8$, P-value $=0.022)$ were the risk factors for the prolonged hospital stay of COVID-19 patients.

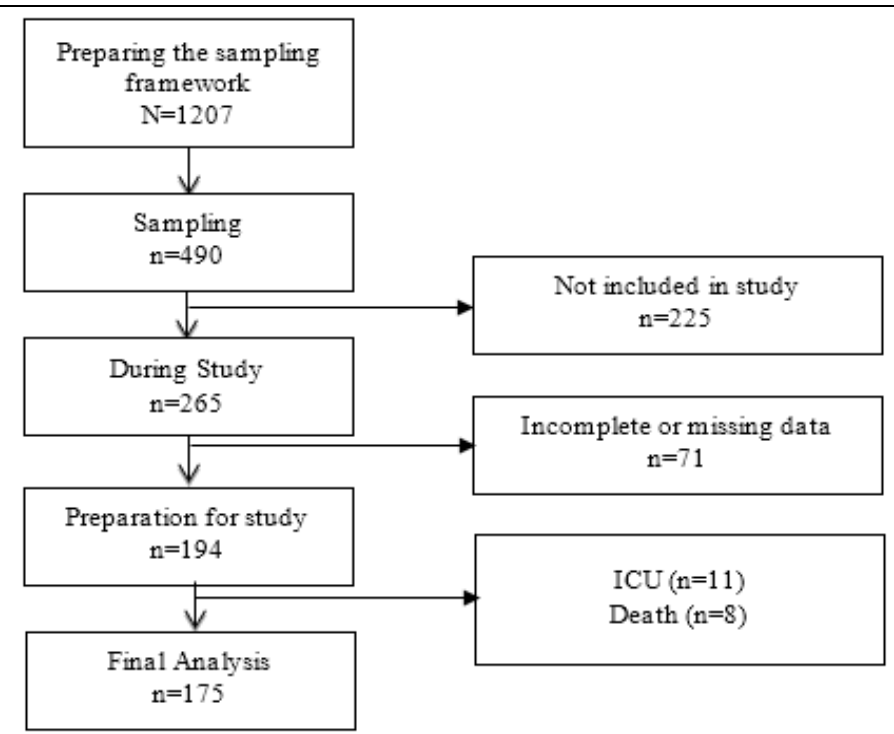

Figure 1. Study diagram of four treatment groups

\begin{tabular}{|c|c|c|c|c|c|c|}
\hline \multirow{2}{*}{ Variable } & \multicolumn{4}{|c|}{ Regimen } & \multirow{2}{*}{ Total $(n=175)$} & \multirow{2}{*}{ P-value } \\
\hline & G1(N=44) & G2(N=37) & G3(N=27) & $\mathrm{G} 4(\mathrm{~N}=67)$ & & \\
\hline Gender (Male) & $18(41 \%)$ & $7(19 \%)$ & $15(56 \%)$ & $40(60 \%)$ & $80(46 \%)$ & 0.001 \\
\hline Smoking (yes) & $2(4 \%)$ & $0(0 \%)$ & $2(7 \%)$ & $2(3 \%)$ & $6(3 \%)$ & 0.442 \\
\hline Age & $57.1( \pm 16.7)$ & $61.7( \pm 14.7)$ & $57.9( \pm 14.5)$ & $59.4( \pm 14.6)$ & $58.9( \pm 15.1)$ & 0.603 \\
\hline BMI & $29.5( \pm 4.9)$ & $27.8( \pm 5.1)$ & $29.3( \pm 8.1)$ & $26.9( \pm 3.9)$ & $28.2( \pm 5.4)$ & 0.068 \\
\hline Hypertension (yes) & $10(\% 23)$ & $6(\% 16)$ & $10(\% 37)$ & $21(\% 31)$ & $47(\% 27)$ & 0.201 \\
\hline Diabetes mellitus (yes) & $11(\% 25)$ & $5(\% 14)$ & $7(\% 26)$ & $17(\% 25)$ & $40(\% 23)$ & 0.507 \\
\hline Hypothyroidism (yes) & $1(\% 2)$ & $1(\% 3)$ & $4(\% 15)$ & $2(\% 3)$ & $8(\% 5)$ & 0.052 \\
\hline IHD (yes) & $1(\% 2)$ & $4(\% 11)$ & $3(\% 11)$ & $7(\% 10)$ & $15(\% 9)$ & 0.316 \\
\hline CABG (yes) & $0(\% 0)$ & $0(\% 0)$ & $0(\% 0)$ & $3(\% 4)$ & $3(\% 2)$ & 0.178 \\
\hline HLP (yes) & $2(\% 5)$ & $1(\% 3)$ & $0(\% 0)$ & $2(\% 3)$ & $5(\% 3)$ & 0.740 \\
\hline Sp02 & $89.9( \pm 7.9)$ & $86.7( \pm 7.6)$ & $87( \pm 5.1)$ & $87.9( \pm 6)$ & $88.1( \pm 6.8)$ & 0.184 \\
\hline Temperature & $36.9( \pm 0.8)$ & $37.3( \pm 0.9)$ & $36.7( \pm 0.3)$ & $36.7( \pm 0.8)$ & $36.88( \pm 0.80)$ & 0.001 \\
\hline SBP & $123.7( \pm 15.8)$ & $118.3( \pm 13)$ & $124.7( \pm 18.8)$ & $121( \pm 18.6)$ & $121.6( \pm 16.9)$ & 0.389 \\
\hline DBP & $75.7( \pm 10.6)$ & $76.6( \pm 10)$ & $78( \pm 14.1)$ & $76.1( \pm 12.6)$ & $76.4( \pm 11.77)$ & 0.879 \\
\hline PR & $92.5( \pm 17.1)$ & $94.5( \pm 17.5)$ & $85.7( \pm 13.7)$ & $85.3( \pm 15.8)$ & $89.11( \pm 16.6)$ & 0.015 \\
\hline $\mathbf{R R}$ & $18.6( \pm 3.5)$ & $18.1( \pm 2.4)$ & $18.2( \pm 2.2)$ & $18.8( \pm 2.7)$ & $18.53( \pm 2.81)$ & 0.613 \\
\hline WBC & $10.9( \pm 19.2)$ & $5.7( \pm 2.6)$ & $6.2( \pm 2.7)$ & $7.1( \pm 3.5)$ & $7.4( \pm 9.1)$ & 0.089 \\
\hline Neutrophil & $73.3( \pm 1.1)$ & $71( \pm 12.2)$ & $73.6( \pm 12.3)$ & $71.9( \pm 14.3)$ & $72.4( \pm 12.7)$ & 0.841 \\
\hline Lymphocytecount & $20.7( \pm 9.3)$ & $20.7( \pm 9.3)$ & $20.7( \pm 9.3)$ & $20.7( \pm 9.3)$ & $21.9( \pm 10.7)$ & 0.530 \\
\hline Hemoglobin & $13.7( \pm 1.9)$ & $13.4( \pm 1.7)$ & $14( \pm 1.3)$ & $13.7( \pm 1.9)$ & $13.7( \pm 1.8)$ & 0.647 \\
\hline PLT & $216.3( \pm 73.5)$ & $191.5( \pm 55)$ & $176.6( \pm 52.2)$ & $196.6( \pm 94.4)$ & $197.1( \pm 76.9)$ & 0.198 \\
\hline CRP & $14.8( \pm 12.7)$ & $24.3( \pm 26.9)$ & $26.6( \pm 13.4)$ & $22.4( \pm 15.3)$ & $21.6( \pm 17.3)$ & 0.038 \\
\hline CR & $1.3( \pm 0.8)$ & $1( \pm 0.4)$ & $1.1( \pm 0.4)$ & $1.3( \pm 0.8)$ & $1.2( \pm 0.7)$ & 0.140 \\
\hline
\end{tabular}


Ghazale AH et al.

\begin{tabular}{|c|c|c|c|c|c|}
\hline \multirow{2}{*}{ Post-treatment } & \multicolumn{4}{|c|}{ Group } & \multirow{2}{*}{ P-value } \\
\hline & G1(N=48) & G2(N=39) & G3(N=30) & G4(N=77) & \\
\hline \multicolumn{6}{|l|}{ Discharge Status } \\
\hline Discharge & $44(\% 92)$ & $37(\% 95)$ & $27(\% 90)$ & $67(\% 87)$ & 0.785 \\
\hline ICU & $3(\% 6)$ & $1(\% 2.5)$ & $1(\% 3)$ & $6(\% 8)$ & \\
\hline \multirow[t]{2}{*}{ Death } & $1(\% 2)$ & $1(\% 2.5)$ & $2(\% 7)$ & $4(\% 5)$ & \\
\hline & $\mathrm{G} 1(\mathrm{~N}=44)$ & $\mathrm{G} 2(\mathrm{~N}=37)$ & $\mathrm{G} 3(\mathrm{~N}=27)$ & $\mathrm{G} 4(\mathrm{~N}=67)$ & P-value \\
\hline Hospital Stay & $5.9( \pm 2.4)$ & $8.1( \pm 4.2)$ & $6.3( \pm 1.7)$ & $6.4( \pm 2.9)$ & 0.008 \\
\hline
\end{tabular}

\begin{tabular}{|c|c|c|c|c|c|}
\hline Variables & Group & $\begin{array}{c}\text { Hospitalization } \\
\text { Mean }( \pm \text { SD) }\end{array}$ & $\begin{array}{c}\text { Discharge } \\
\text { Mean }( \pm \text { SD) }\end{array}$ & $\begin{array}{l}\text { P-value } \\
\text { Within }\end{array}$ & $\begin{array}{c}\text { P-value } \\
\text { Between }\end{array}$ \\
\hline \multirow{4}{*}{ Hemoglobin } & G1 & $13.69( \pm 1.94)$ & $12.41( \pm 2.15)$ & $<0.001$ & \multirow{4}{*}{0.527} \\
\hline & G2 & $13.43( \pm 1.68)$ & $10.57( \pm 1.7)$ & 0.018 & \\
\hline & G3 & $14.03( \pm 1.34)$ & $13.44( \pm 1.83)$ & 0.011 & \\
\hline & G4 & $13.72( \pm 1.93)$ & $13.16( \pm 2.33)$ & 0.003 & \\
\hline \multirow{4}{*}{ PLT } & G1 & $209.24( \pm 73.76)$ & $252.56( \pm 85.18)$ & 0.002 & \multirow{4}{*}{0.022} \\
\hline & G2 & $189.73( \pm 55.3)$ & $253( \pm 98.17)$ & 0.001 & \\
\hline & G3 & $180.9( \pm 55.53)$ & $207.3( \pm 72.94)$ & 0.01 & \\
\hline & G4 & $195.83( \pm 92.12)$ & $222.32( \pm 100.44)$ & 0.04 & \\
\hline \multirow{4}{*}{ CRP } & G1 & $14.83( \pm 12.67)$ & $12.15( \pm 10.83)$ & 0.348 & \multirow{4}{*}{0.275} \\
\hline & G2 & $24.31( \pm 26.86)$ & $20.54( \pm 7.72)$ & 0.225 & \\
\hline & G3 & $26.56( \pm 13.41)$ & $26.43( \pm 15.45)$ & 0.845 & \\
\hline & G4 & $22.38( \pm 15.31)$ & $27.93( \pm 14.53)$ & 0.258 & \\
\hline \multirow{4}{*}{ Creatinine } & G1 & $1.34( \pm 0.8)$ & $1.39( \pm 0.84)$ & 0.309 & \multirow{4}{*}{0.829} \\
\hline & G2 & $0.97( \pm 0.41)$ & $0.8( \pm 0.14)$ & 0.157 & \\
\hline & G3 & $1.14( \pm 0.35)$ & $0.96( \pm 0.19)$ & 0.022 & \\
\hline & G4 & $1.25( \pm 0.8)$ & $1.15( \pm 0.48)$ & 0.009 & \\
\hline \multirow{4}{*}{ WBC } & G1 & $10.91( \pm 19.22)$ & $19.04( \pm 34.82)$ & 0.032 & \multirow{4}{*}{0.008} \\
\hline & G2 & $5.73( \pm 2.62)$ & $6.7( \pm 1.04)$ & 0.109 & \\
\hline & G3 & $6.24( \pm 2.66)$ & $6.37( \pm 2.3)$ & 0.936 & \\
\hline & G4 & $7.12( \pm 3.51)$ & $6.13( \pm 1.77)$ & 0.083 & \\
\hline \multirow{4}{*}{ Neutrophil } & G1 & $73.29( \pm 11.1)$ & $71.17( \pm 13.22)$ & 0.077 & \multirow{4}{*}{0.433} \\
\hline & G2 & $70.96( \pm 12.24)$ & $71.2( \pm 13.2)$ & 0.881 & \\
\hline & G3 & $73.56( \pm 12.28)$ & $71.17( \pm 12.89)$ & 0.353 & \\
\hline & G4 & $71.85( \pm 14.27)$ & $72.76( \pm 11.25)$ & 0.469 & \\
\hline \multirow{4}{*}{ Lymphocyte } & G1 & $20.68( \pm 9.34)$ & $21.22( \pm 11.7)$ & 0.904 & \multirow{4}{*}{0.245} \\
\hline & G2 & $23.49( \pm 10.11)$ & $12.5( \pm 4.1)$ & 0.180 & \\
\hline & G3 & $20.07( \pm 9.3)$ & $23.44( \pm 10.46)$ & 0.343 & \\
\hline & G4 & $22.45( \pm 12.3)$ & $22.5( \pm 11.52)$ & 0.173 & \\
\hline \multirow{4}{*}{ Sp02 } & G1 & $89.89( \pm 7.94)$ & $93.17( \pm 3.69)$ & 0.004 & \multirow{4}{*}{0.131} \\
\hline & G2 & $86.72( \pm 7.57)$ & $88.86( \pm 17.92)$ & 0.001 & \\
\hline & G3 & $86.96( \pm 5.13)$ & $90.39( \pm 3.05)$ & $<0.001$ & \\
\hline & G4 & $87.89( \pm 6.01)$ & $90.81( \pm 4.95)$ & 0.001 & \\
\hline \multirow{4}{*}{ Temperature } & G1 & $36.88( \pm 0.79)$ & $36.64( \pm 0.41)$ & 0.029 & \multirow{4}{*}{0.014} \\
\hline & G2 & $37.31( \pm 0.91)$ & $36.75( \pm 0.4)$ & 0.039 & \\
\hline & G3 & $36.68( \pm 0.35)$ & $36.57( \pm 0.4)$ & 0.155 & \\
\hline & G4 & $36.71( \pm 0.8)$ & $36.63( \pm 0.6)$ & 0.355 & \\
\hline \multirow{4}{*}{ SBP } & G1 & $123.66( \pm 15.8)$ & $120.85( \pm 11.9)$ & 0.580 & \multirow{4}{*}{0.468} \\
\hline & G2 & $118.32( \pm 12.97)$ & $121.33( \pm 12.46)$ & 0.410 & \\
\hline & G3 & $124.7( \pm 18.82)$ & $122.08( \pm 11.97)$ & 0.757 & \\
\hline & G4 & $121( \pm 18.63)$ & $118.84( \pm 15.83)$ & 0.241 & \\
\hline & G1 & $75.68( \pm 10.61)$ & $77.88( \pm 8.28)$ & 0.120 & \\
\hline DRP & G2 & $76.59( \pm 10)$ & $74.38( \pm 7.27)$ & 0.944 & \\
\hline DBP & G3 & $77.96( \pm 14.08)$ & $80.17( \pm 7.48)$ & 0.430 & 0.128 \\
\hline & G4 & $76.13( \pm 12.6)$ & $74.4( \pm 10.55)$ & 0.166 & \\
\hline & G1 & $92.52( \pm 17.09)$ & $81.81( \pm 10.86)$ & 0.002 & \\
\hline & G2 & $94.54( \pm 17.47)$ & $82.94( \pm 13.93)$ & 0.05 & \\
\hline PR & G3 & $85.7( \pm 13.75)$ & $78.79( \pm 12.32)$ & 0.028 & 0.479 \\
\hline & G4 & $85.28( \pm 15.79)$ & $80.6( \pm 11.28)$ & 0.054 & \\
\hline & G1 & $18.64( \pm 3.51)$ & $18.41( \pm 1.78)$ & 0.744 & \\
\hline & G2 & $18.14( \pm 2.39)$ & $17.07( \pm 1.79)$ & 0.153 & \\
\hline RR & G3 & $18.19( \pm 2.22)$ & $18.35( \pm 3.97)$ & 0.493 & 0.323 \\
\hline & G4 & $18.83( \pm 2.73)$ & $18.72( \pm 2)$ & 0.945 & \\
\hline
\end{tabular}

\section{Discussion}

The COVID-19 pandemic has had a notable impact on the death rate of the health system and inflicted huge socio-economic costs on numerous countries around the world over the past year (15). The lack of a clear, reliable, and proven standard therapy for COVID-19 is one of the serious public health 
problems. Therefore, the current research aimed to evaluate the effect of combination therapy on the treatment of COVID-19 disease. Based on the obtained result in this study, the combination of azithromycin, prednisolone, and naproxen led to better clinical results, compared to the other three combination therapy regimens. This result can be attributed to the concurrent use of azithromycin and prednisolone. Moreover, an early upper respiratory tract shedding of the virus has been confirmed in asymptomatic and pauci symptomatic patients at the early emergence of the symptoms (16). Therefore, antiviral regimens and handling of virus load lost effect in symptomatic patients over time, considering the fact that patients in this study were not in the early phase of the disease. Moreover, antiinflammatory drugs have been recommended for the treatment of pulmonary lesions and improvement of clinical outcomes in some patients due to their effectiveness in controlling cytokine storms (17).

The results of a study conducted by Russell indicated that low-dose prednisolone could have beneficial effects on the treatment of COVID-19 (18). Another study performed by Arshad et al. showed that the application of azithromycin was associated with a reduction in mortality rate associated with COVID-19 (19). In addition, a review study conducted by Jean et al. reported the excellent clinical efficacy of hydroxychloroquine along with azithromycin on COVID-19 patients (20). The pharmacological mechanism of azithromycin and prednisolone in the treatment of COVID-19 is presented in the studies conducted by Sultana et al. (21) and Caruso et al., respectively (22). Based on the results of a study conducted by Vahedi et al. low-dose prednisolone in combination with azithromycin, naproxen, and lopinavir/ritonavir led to a reduction in COVID-19 hospital stay (11). Based on the results of a metaanalysis conducted by Sarma et al. (23) no significant improvement was observed in the status of COVID-19 patients treated with hydroxychloroquine. In addition, the results of a review study by Yousefi et al. indicated that neuraminidase inhibitors, such as oseltamivir, peramivir, and zanamivir were not recommended for the treatment of COVID-19 disease (24).

The results of another study showed that laboratory variables, such as WBC, lymphocyte, CRP, as well as SPO2 were associated with a hospital mortality rate (25).

Regarding the limitations of the present study, one can refer to the lack of certain clinical recording and laboratory results, which was due to inadequate patient profiles in the hospitals' electronic medical reports. Consequently, the recommendations in this study were made based on the available data.

\section{Conclusion}

Based on the obtained result in this study, the combination of azithromycin, prednisolone, and naproxen was a more effective treatment regimen, compared to the other three combination therapy regimens.

\section{Acknowledgments}

The authors gratefully acknowledge financial support from Baqiyatallah University of Medical Sciences and would like to thank all the personnel, especially the staff of Baqiyatallah Hospital, for their cooperation throughout the study.

\section{Footnotes}

Authors' contribution: Amir Hosein Ghazale, Ali Ghazvini and Mostafa Ghanei conducted the study design. Data collection was performed by Ensieh Vahedi, Shideh Omidian, Abolfazl Mozafari, Mohammad Rezapour, Nafiseh Rastgoo, Fatemeh Movaseghi, Fateme Mansouri, Mohammad Ali Zohal, Maryam Gheraati, Hassan Goodarzi, Amir Mohammad Dehghan Banadkooki, Amir Mohammad Dehghan Banadkooki, Shahrzad Saloo, and Hesamodin Salou. Data analysis was carried out by Mohammad Gholami Fesharaki. Eventually, Mohammad Gholami Fesharaki, Mostafa Ghanei, and Amir Hosein Ghazale wrote the manuscript.

Ethical Approval: The cohort study was approved by the Medical Ethics Committee of Baqiyatallah University of Medical Sciences (IR.BMSU.REC. 1399.001) and was conducted in accordance with the ethical standards as laid down in the 1964 Declaration of Helsinki and its later amendments.

Conflict of interests: The authors declare that he has no conflicts of interest regarding the publication of the present study.

Funding/Support: This study was funded by Baqiyatallah University of Medical Sciences.

Informed consent: Informed consent was not required to conduct this study.

\section{References}

1. World Health Organization. WHO characterizes COVID-19 as a pandemic. Geneva: World Health Organization; 2020.

2. Cao B, Wang Y, Wen D, Liu W, Wang J, Fan G, et al. A trial of lopinavir-ritonavir in adults hospitalized with severe Covid19. N Engl J Med. 2020;382(19):1787-99. doi: 10.1056/ NEJMoa2001282. [PubMed: 32187464].

3. Al-Tawfiq JA, Al-Homoud AH, Memish ZA. Remdesivir as a possible therapeutic option for the COVID-19. Travel Med Infect Dis. 2020;34:101615. doi: 10.1016/j.tmaid.2020.101615. [PubMed: 32145386].

4. Elfiky AA. Anti-HCV, nucleotide inhibitors, repurposing against COVID-19. Life Sci. 2020;248:117477. doi: 10.1016/j.lfs. 2020.117477. [PubMed: 32119961].

5. Mitjà 0 , Clotet $\mathrm{B}$. Use of antiviral drugs to reduce COVID-19 transmission. Lancet Glob Health. 2020;8(5):e639-40. doi: 10.1016/S2214-109X(20)30114-5. [PubMed: 32199468].

6. Cortegiani A, Ingoglia G, Ippolito M, Giarratano A, Einav S. A systematic review on the efficacy and safety of chloroquine for the treatment of COVID-19. J Crit Care. 2020;57:279-83. doi: 
10.1016/j.jcrc.2020.03.005. [PubMed: 32173110].

7. Sahu P, Galhotra A, Raj U, Ranjan RV. A study of self-reported health problems of the people living near railway tracks in Raipur city. J Family Med Prim Care. 2020;9(2):740-4. doi: 10.4103/jfmpc.jfmpc_1029_19. [PubMed: 32318412].

8. Yang Z, Liu J, Zhou Y, Zhao X, Zhao Q, Liu J. The effect of corticosteroid treatment on patients with coronavirus infection: a systematic review and meta-analysis. J Infect. 2020;81(1):e13-20. doi: 10.1016/j.jinf.2020.03.062. [PubMed: 32283144].

9. Zha L, Li S, Pan L, Tefsen B, Li Y, French N, et al. Corticosteroid treatment of patients with coronavirus disease 2019 (COVID-19). Med J Aust. 2020;212(9):416-20. doi: 10.5694/mja2.50577. [PubMed: 32266987].

10. Russell CD, Millar JE, Baillie JK. Clinical evidence does not support corticosteroid treatment for 2019-nCoV lung injury. Lancet. 2020;395(10223):473-5. doi: 10.1016/S01406736(20)30317-2. [PubMed: 32043983].

11. Vahedi E, Ghanei M, Ghazvini A, Azadi H, Izadi M, Panahi Y, et al. The clinical value of two combination regimens in the Management of Patients Suffering from Covid-19 pneumonia: a single centered, retrospective, observational study. Daru. 2020;28(2):507-16. doi: 10.1007/s40199-020-00353-w. [PubMed: 32562159].2020;212(9):416-20. doi: 10.5694/mja2. 50577. [PubMed: 32266987].

12. Huang C, Wang Y, Li X, Ren L, Zhao J, Hu Y, et al. Clinical features of patients infected with 2019 novel coronavirus in Wuhan, China. Lancet. 2020;395(10223):497-506. doi: 10.1016/S0140-6736(20)30183-5. [PubMed: 31986264].

13. Guo YR, Cao QD, Hong ZS, Tan YY, Chen SD, Jin HJ, et al. The origin, transmission and clinical therapies on coronavirus disease 2019 (COVID-19) outbreak-an update on the status. Mil Med Res. 2020;7(1):11. doi: 10.1186/s40779-020-002400. [PubMed: 32169119].

14. Shi H, Han X, Jiang N, Cao Y, Alwalid O, Gu J, et al. Radiological findings from 81 patients with COVID-19 pneumonia in Wuhan, China: a descriptive study. Lancet Infect Dis. 2020;20(4):425-34. doi: 10.1016/S1473-3099(20)30086-4. [PubMed: 32105637].

15. Najafi A, Ghanei M, Janbabaei G, Velayati AA, Saadat SH, Jamaati $\mathrm{H}$, et al. Real clinical practice and therapeutic management following COVID-19 crisis in two hospitals in Iran: a statistical and conceptual view. Tanaffos. 2020;19(2):112-21. [PubMed: 33262798].

16. Zou L, Ruan F, Huang M, Liang L, Huang H, Hong Z, et al. SARSCoV-2 viral load in upper respiratory specimens of infected patients. N Engl J Med. 2020;382(12):1177-9. doi: 10.1056/NEJMc2001737. [PubMed: 32074444].

17. Ma J, Xia P, Zhou Y, Liu Z, Zhou X, Wang J, et al. Potential effect of blood purification therapy in reducing cytokine storm as a late complication of critically ill COVID-19. Clin Immunol. 2020;214:108408. doi: 10.1016/j.clim.2020.108408. [PubMed: 32247038].

18. Russell B, Moss C, George G, Santaolalla A, Cope A, Papa S, et al. Associations between immune-suppressive and stimulating drugs and novel COVID-19-a systematic review of current evidence. Ecancermedicalscience. 2020;14(1022). doi: 10.3332/ecancer.2020.1022. [PubMed: 32256705].

19. Arshad S, Kilgore P, Chaudhry ZS, Jacobsen G, Wang DD, Huitsing $\mathrm{K}$, et al. Treatment with hydroxychloroquine, azithromycin, and combination in patients hospitalized with COVID-19. Int J Infect Dis. 2020;97:396-403. doi: 10.1016/j.ijid.2020.06.099. [PubMed: 32623082].

20. Jean SS, Lee PI, Hsueh PR. Treatment options for COVID-19: The reality and challenges. J Microbiol Immunol Infect. 2020;53(3):436-43. doi: 10.1016/j.jmii.2020.03.034. [PubMed: 32307245].

21. Sultana J, Cutroneo PM, Crisafulli S, Puglisi G, Caramori G, Trifirò G. Azithromycin in COVID-19 patients: pharmacological mechanism, clinical evidence and prescribing guidelines. Drug Saf. 2020;43(8):691-8. doi: 10.1007/s40264-020-00976-7. [PubMed: 32696429].

22. Caruso F, Rossi M, Pedersen JZ, Incerpi S. Computational studies reveal mechanism by which quinone derivatives can inhibit SARS-CoV-2. Study of embelin and two therapeutic compounds of interest, methyl prednisolone and dexamethasone. J Infect Public Health. 2020;13(12):1868-77. doi: 10.1016/j.jiph.2020.09.015. [PubMed: 33109497].

23. Sarma P, Kaur H, Kumar H, Mahendru D, Avti P, Bhattacharyya A, et al. Virological and clinical cure in COVID-19 patients treated with hydroxychloroquine: A systematic review and meta-analysis. J Med Virol. 2020;92(7):776-85. doi: 10.1002/jmv.25898. [PubMed: 32297988].

24. Yousefi B, Valizadeh S, Ghaffari H, Vahedi A, Karbalaei M Eslami M. A global treatments for coronaviruses including COVID-19. J Cell Physiol. 2020;235(12):9133-42. doi: 10.1002/jcp.29785. [PubMed: 32394467].

25. Ali H, Daoud A, Mohamed MM, Salim SA, Yessayan L, Baharani J, et al. Survival rate in acute kidney injury superimposed COVID-19 patients: a systematic review and meta-analysis. Ren Fail. 2020;42(1):393-7. doi: 10.1080/0886022X.2020.1756323. [PubMed: 32340507]. 